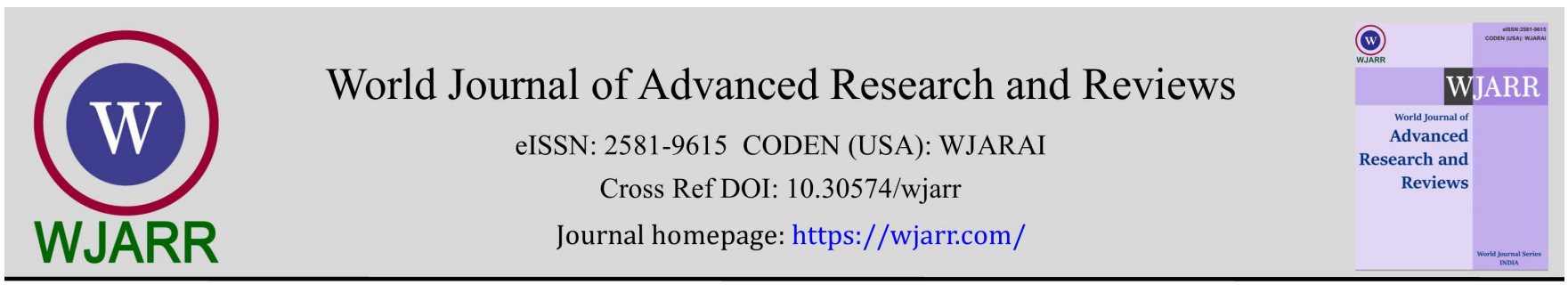

(REVIEW ARTICLE)

\title{
Basic concepts in clinical immunology: A review
}

\author{
Andrew Nakibinge Kiboneka * \\ Department of Paediatrics' Case Hospital Kampala, Uganda.
}

World Journal of Advanced Research and Reviews, 2021, 12(03), 490-496

Publication history: Received on 15 November 2021; revised on 18 December 2021; accepted on 20 December 2021

Article DOI: https://doi.org/10.30574/wjarr.2021.12.3.0745

\begin{abstract}
Immunity is the state of protection against foreign pathogens or substances(antigens). Host defence mechanisms consist of innate immunity (natural immunity), which mediates the initial protection against infections, and adaptive immunity (specific/acquired immunity), which develops more slowly and provides more specialized and more effective defence against infections. The immune system evolved to protect multicellular organisms against pathogens.

The body is protected against pathogens by a variety of effector cells and molecules that together make up the immune system. All the cellular elements of blood, including the red blood cells, platelets and white blood cells of the immune system, ultimately derive from the hematopoietic stem cells of the bone marrow. The cells of the adaptive immune system consist of lymphocytes, antigen-presenting cells and effector cells that eliminate microbes.

Strategies of avoidance, and tolerance represent different ways of dealing with pathogens. Anatomic barriers and chemical barriers e.g complement and antimicrobial proteins may be considered as primary forms of avoidance.Macrophages,neutrophils and dendritic cells are important cells that that detect infection. Pattern recognition receptors (PRRs) recognize simple molecules and regular patterns of molecular structures called pathogen associated molecular patterns. Some PRR are transmembrane proteins e.g Toll like receptors (TLRs). Vaccination is a simple, safe, and effective way of protecting people against harmful diseases, before they come into contact with them. Immunization is the process whereby a person is made immune or resistant to an infectious disease, typically by the administration of a vaccine.
\end{abstract}

Inappropriate immune responses can result into hypersensitivity, autoimmune disease or immune deficiency.

Keywords: Immunology; Innate and adaptive immunity; Complement.

\section{Introduction}

Immunology is a relatively new science. Its origin is usually attributed to Edward Jenner who discovered in 1796 that cowpox, or vaccinia, induced protection against human smallpox, an often fatal disease. Jenner called his procedure vaccination, and this term is still used to describe the inoculation of healthy individuals with weakened or attenuated strains of disease-causing agents to provide protection from disease. Although Jenner's bold experiment was successful, it took almost two centuries for smallpox vaccination to become universal, an advance that enabled the World Health Organization to announce in 1979 that smallpox had been eradicated arguably the greatest triumph of modern medicine [1].

The eradication of smallpox by vaccination. After a period of 3 years in which no cases of smallpox were recorded, the World Health Organization was able to announce in 1979 that smallpox had been eradicated [1].

\footnotetext{
* Corresponding author: Andrew Kiboneka

Department of Paediatrics' Case Hospital Kampala, Uganda.

Copyright (C) 2021 Author(s) retain the copyright of this article. This article is published under the terms of the Creative Commons Attribution Liscense 4.0.
} 
When Jenner introduced vaccination, he knew nothing of the infectious agents that cause disease: it was not until late in the 19th century that Robert Koch proved that infectious diseases are caused by microorganisms, each one responsible for a particular disease, or pathology.[2,3]. We now recognize four broad categories of disease-causing microorganisms, or pathogens: these are viruses, bacteria, pathogenic fungi, and other relatively large and complex eukaryotic organisms collectively termed parasites.[4].

The discoveries of Koch and other great 19th century microbiologists stimulated the extension of Jenner's strategy of vaccination to other diseases. In the 1880s, Louis Pasteur devised a vaccine against cholera in chickens, and developed a rabies vaccine that proved a spectacular success upon its first trial in a boy bitten by a rabid dog. These practical triumphs led to a search for the mechanism of protection and to the development of the science of immunology. In 1890, Emil von Behring and Shibasaburo Kitasato discovered that the serum of vaccinated individuals contained substanceswhich they called antibodies - that specifically bound to the relevant pathogen.(5)

A specific immune response, such as the production of antibodies against a particular pathogen, is known as an adaptive immune response, because it occurs during the lifetime of an individual as an adaptation to infection with that pathogen. In many cases, an adaptive immune response confers lifelong protective immunity to reinfection with the same pathogen. This distinguishes such responses from innate immunity, which, at the time that von Behring and Kitasato discovered antibodies, was known chiefly through the work of the great Russian immunologist Elie Metchnikoff. Metchnikoff discovered that many microorganisms could be engulfed and digested by phagocytic cells, which he called macrophages. These cells are immediately available to combat a wide range of pathogens without requiring prior exposure and are a key component of the innate immune system. Antibodies, by contrast, are produced only after infection, and are specific for the infecting pathogen. The antibodies present in a given person therefore directly reflect the infections to which he or she has been exposed.[6].

Indeed, it quickly became clear that specific antibodies can be induced against a vast range of substances. Such substances are known as antigens because they can stimulate the generation of antibodies. We shall see, however, that not all adaptive immune responses entail the production of antibodies, and the term antigen is now used in a broader sense to describe any substance that can be recognized by the adaptive immune system.

Both innate immunity and adaptive immune responses depend upon the activities of white blood cells, or leukocytes. Innate immunity largely involves granulocytes and macrophages. Granulocytes, also called polymorphonuclear leukocytes, are a diverse collection of white blood cells whose prominent granules give them their characteristic staining patterns; they include the neutrophils, which are phagocytic. The macrophages of humans and other vertebrates are presumed to be the direct evolutionary descendants of the phagocytic cells present in simpler animals, such as those that Metchnikoff observed in sea stars. Adaptive immune responses depend upon lymphocytes, which provide the lifelong immunity that can follow exposure to disease or vaccination. The innate and adaptive immune systems together provide a remarkably effective defense system. It ensures that although we spend our lives surrounded by potentially pathogenic microorganisms, we become ill only relatively rarely. Many infections are handled successfully by the innate immune system and cause no disease; others that cannot be resolved by innate immunity trigger adaptive immunity and are then overcome successfully, followed by lasting immunological memory.[6].

The main focus of this review will be on the diverse mechanisms of innate \&adaptive immunity.

\section{Methodology}

A systematic review on PUBMed and internationally recognized textbooks of immunology between January 2015 thru October 2021.

\section{Results}

\subsection{The components of the immune system}

The immune system is the body's biological defense system. The main purpose of the immune system is to identify self from non-self. The immune system identifies and defends the body from non-self-proteins, viruses, bacteria, fungi, parasites and other pathogens. Occasionally, the immune system can make a mistake and attack itself, resulting in autoimmune disorders. 
The immune system comprises many different cells, organs, and tissues that work together to combat infection, cellular damage and disease. Cells of the immune system include white blood cells, such as macrophages, as well as T and B lymphocytes. The main lymphoid tissues of the immune system are the thymus and the bone marrow. White blood cells and platelets constitutes immune cells.

- The immune system consists of many parts that work together to defend the body against invaders. The primary parts of the immune system include the bone marrow and thymus. The bone marrow is extremely important to the immune system because all the body's blood cells (including T and B lymphocytes) originate in the bone marrow. B lymphocytes remain in the marrow to mature, while T lymphocytes travel to the thymus.

The thymus is a bi-lobed gland located above the heart, behind the sternum and between the lungs. The thymus is only active through puberty, then it slowly shrinks and is replaced by fat and connective tissue. The thymus is responsible for producing the hormone thymosin, which in turn aids in the production of $\mathrm{T}$ cells. While in the thymus, $\mathrm{T}$ cells multiply, acquire different antigen receptors, and differentiate into helper $\mathrm{T}$ cells and cytotoxic $\mathrm{T}$ cells. Various proteins (e.g., CD4, CD8) are expressed on the T cell surface. The thymus will have produced all the T cells an individual needs by puberty [7].

After the T and B lymphocytes have matured in the thymus and bone marrow, they then travel to the lymph nodes and spleen where they remain until the immune system is activated. Lymph nodes are located throughout the body. The spleen is located in the upper left area of the abdomen, behind the stomach, and under the diaphragm. The main function of the spleen is to filter the blood. Healthy red blood cells easily pass through the spleen; however, damaged red blood cells are broken down by macrophages (large white blood cells specialized in engulfing and digesting cellular debris, pathogens and other foreign substances in the body) in the spleen. The spleen serves as a storage unit for platelets and white blood cells. The spleen aids the immune system by identifying microorganisms that may cause infection. [8].

In addition to the lymph nodes and spleen, mucosal associated lymphoid tissues (MALTs) and gut associated lymphoid tissues (GALTs) play a vital role in the immune system, although they are considered to be part of the lymphatic system. MALTs are lymphoid tissues found in parts of the body where mucosa is present, such as the intestines, eyes, nose, skin and mouth. They contain lymphocytes and macrophages that defend against pathogens attempting to enter from outside the body. GALTs are lymphoid tissues found in the mucosa and submucosa of the gastrointestinal tract, tonsils, appendix and Peyer's patches in the small intestine.[8].

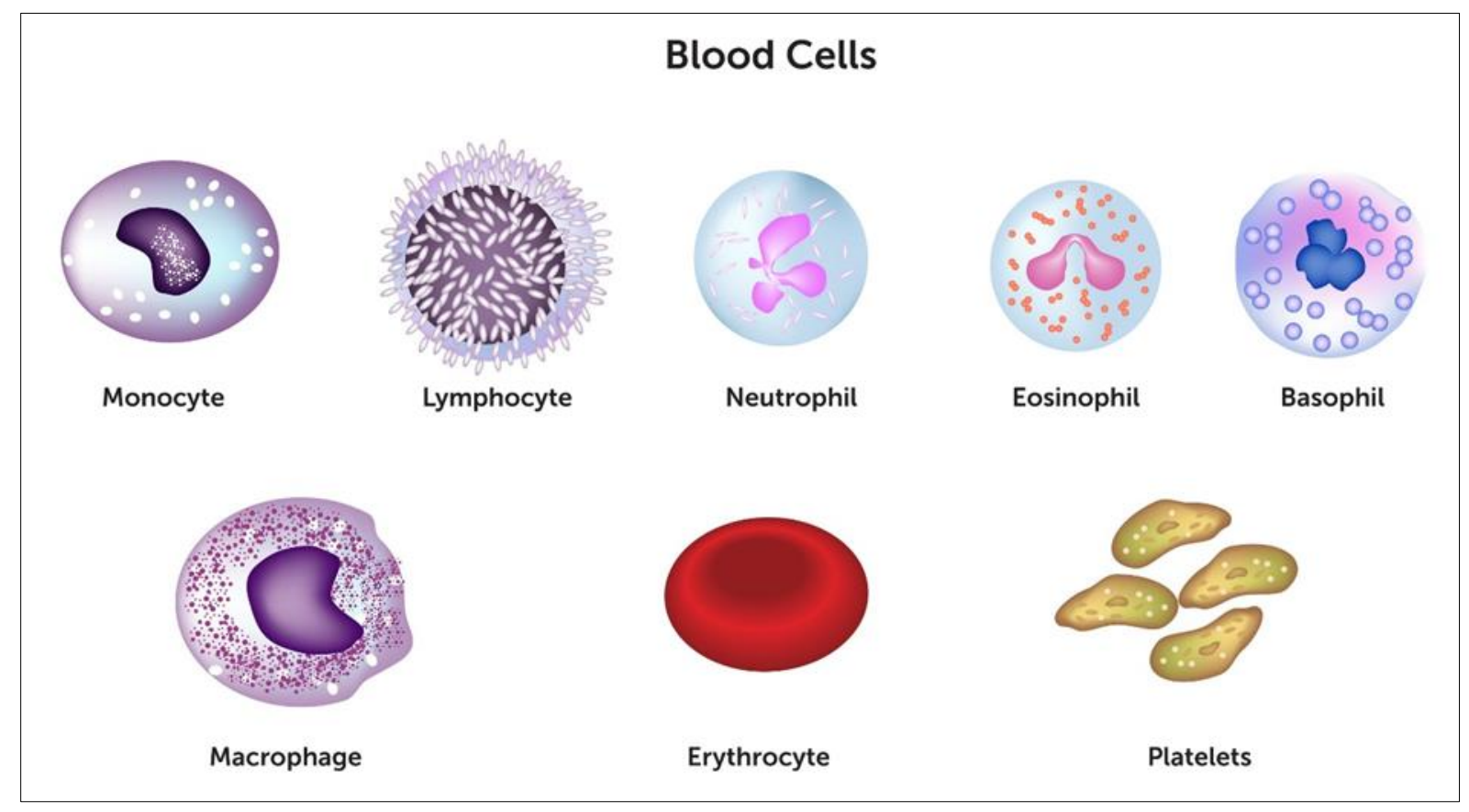

Figure 1 Blood cells 


\subsection{Principles of innate and adaptive immunity}

- The first line of defence against non-self-pathogens is the innate, or non-specific, immune response. The innate immune response consists of physical, chemical and cellular defences against pathogens. ... Adaptive immunity is also referred to as acquired immunity or specific immunity and is only found in vertebrates.

- The immune system is typically divided into two categories--innate and adaptive--although these distinctions are not mutually exclusive.[9].

\subsection{Innate immunity}

Innate immunity refers to nonspecific defense mechanisms that come into play immediately or within hours of an antigen's appearance in the body. These mechanisms include physical barriers such as skin, chemicals in the blood, and immune system cells that attack foreign cells in the body. The innate immune response is activated by chemical properties of the antigen.

\subsection{Adaptive immunity}

Adaptive immunity refers to antigen-specific immune response. The adaptive immune response is more complex than the innate. The antigen first must be processed and recognized. Once an antigen has been recognized, the adaptive immune system creates an army of immune cells specifically designed to attack that antigen. Adaptive immunity also includes a "memory" that makes future responses against a specific antigen more efficient.

\subsection{Self vs. Non-self: How does the body know?}

In order to be effective, the immune system needs to be able to identify which particles are foreign, and which are a part of your body. Let's define some terms before we jump in to how this works:

- Self refers to particles, such as proteins and other molecules that are a part of, or made by, your body. They can be found circulating in your blood or attached to different tissues. Something that is self should not be targeted and destroyed by the immune system. The non-reactivity of the immune system to self-particles is called tolerance.

- Non-self refers to particles that are not made by your body, and are recognized as potentially harmful. These are sometimes called foreign bodies. Non-self-particles or bodies can be bacteria, viruses, parasites, pollen, dust, and toxic chemicals. The non-self-particles and foreign bodies that are infectious or pathogenic, like bacteria, viruses, and parasites, make proteins called antigens that allow the human body to know that they intend to cause damage.

- Antigens are anything that causes an immune response. Antigens can be entire pathogens, like bacteria, viruses, fungi, and parasites, or smaller proteins that pathogens express. Antigens are like a name tag for each pathogen that announce the pathogens' presence to your immune system. Some pathogens are general, whereas others are very specific. A general antigen would announce "I'm dangerous", whereas a specific antigen would announce "I'm a bacteria that will cause an infection in your gastrointestinal tract" or "I'm the influenza virus".

- Cytokines are molecules that are used for cell signaling, or cell-to-cell communication. Cytokines are similar to chemokines, wherein they can be used to communicate with neighboring or distant cells about initiating an immune response. Cytokines are also used to trigger cell trafficking, or movement, to a specific area of the body.

- Chemokines are a type of cytokines that are released by infected cells. Infected host cells release chemokines in order to initiate an immune response, and to warn neighboring cells of the threat.[10].

\subsection{Innate Immune System}

The innate immune system is made of defenses against infection that can be activated immediately once a pathogen attacks. The innate immune system is essentially made up of barriers that aim to keep viruses, bacteria, parasites, and other foreign particles out of your body or limit their ability to spread and move throughout the body. The innate immune system includes:

- $\quad$ Physical Barriers

- $\quad$ such as skin, the gastrointestinal tract, the respiratory tract, the nasopharynx, cilia, eyelashes and other body hair.

- Defense Mechanisms

- $\quad$ such as secretions, mucous, bile, gastric acid, saliva, tears, and sweat.

- General Immune Responses 
- $\quad$ such as inflammation, complement, and non-specific cellular responses. The inflammatory response actively brings immune cells to the site of an infection by increasing blood flow to the area. Complement is an immune response that marks pathogens for destruction and makes holes in the cell membrane of the pathogen.

The innate immune system is always general, or nonspecific, meaning anything that is identified as foreign or non-self is a target for the innate immune response. The innate immune system is activated by the presence of antigens and their chemical properties.

The bone marrow of an average healthy adult makes approximately 100 billion new neutrophils per day.

Neutrophils are typically the first cells to arrive at the site of an infection because there are so many of them in circulation at any given time.

- $\quad$ Eosinophils: Eosinophils are granulocytes target multicellular parasites. Eosinophils secrete a range of highly toxic proteins and free radicals that kill bacteria and parasites. The use of toxic proteins and free radicals also causes tissue damage during allergic reactions, so activation and toxin release by eosinophils is highly regulated to prevent any unnecessary tissue damage.

- While eosinophils only make up 1-6\% of the white blood cells, they are found in many locations, including the thymus, lower gastrointestinal tract, ovaries, uterus, spleen, and lymph nodes.

- Basophils: Basophils are also granulocytes that attack multicellular parasites. Basophils release histamine, much like mast cells. The use of histamine makes basophils and mast cells key players in mounting an allergic response.

- $\quad$ Natural Killer cells: Natural Killer cells (NK cells), do not attack pathogens directly. Instead, natural killer cells destroy infected host cells in order to stop the spread of an infection. Infected or compromised host cells can signal natural kill cells for destruction through the expression of specific receptors and antigen presentation.

- Dendritic cells: Dendritic cells are antigen-presenting cells that are located in tissues, and can contact external environments through the skin, the inner mucosal lining of the nose, lungs, stomach, and intestines. Since dendritic cells are located in tissues that are common points for initial infection, they can identify threats and act as messengers for the rest of the immune system by antigen presentation. Dendritic cells also act as bridge between the innate immune system and the adaptive immune system.

\section{The Adaptive Immune System}

- The adaptive immune response provides the vertebrate immune system with the ability to recognize and remember specific pathogens to generate immunity, and mount stronger attacks each time the pathogen is encountered.

- $\quad$ The cells of the adaptive immune system are a type of leukocyte called a lymphocyte. B cells and T cells are the major types of lymphocytes involved in adaptive immunity.

- $\quad$ B and T cells can create memory cells to defend against future attacks by the same pathogen by mounting a stronger and faster adaptive immune response against that pathogen before it can even cause symptoms of infection.

- $\quad$ Antigen -presenting cells present captured antigens to immature lymphocytes, which then mature to be specific to that antigen and work to destroy pathogens that express that antigen.

- Hypersensitivity disorders (allergies) may occur when an adaptive immune response forms against antigens that aren't associated with pathogens, such as pollen. More complex hypersensitivity disorders may involve cytotoxic T cells and cause chronic inflammation and damage to the body's own tissues.

\subsection{The Complement System}

The complement system (also called the complement cascade) is a mechanism that complements other aspects of the immune response. Typically, the complement system acts as a part of the innate immune system, but it can work with the adaptive immune system if necessary.

The complement system is made of a variety of proteins that, when inactive, circulate in the blood. When activated, these proteins come together to initiate the complement cascade, which starts the following steps:[11]. 


\subsection{Opsonization}

Opsonization is a process in which foreign particles are marked for phagocytosis. All of the pathways require an antigen to signal that there is a threat present. Opsonization tags infected cells and identifies circulating pathogens expressing the same antigens.

- $\quad$ Chemotaxis: Chemotaxis is the attraction and movement of macrophages to a chemical signal. Chemotaxis uses cytokines and chemokines to attract macrophages and neutrophils to the site of infection, ensuring that pathogens in the area will be destroyed. By bringing immune cells to an area with identified pathogens, it improves the likelihood that the threats will be destroyed and the infection will be treated.

- $\quad$ Cell Lysis: Lysis is the breaking down or destruction of the membrane of a cell. The proteins of the complement system puncture the membranes of foreign cells, destroying the integrity of the pathogen. Destroying the membrane of foreign cells or pathogens weakens their ability to proliferate, and helps to stop the spread of infection.

- Agglutination: Agglutination uses antibodies to cluster and bind pathogens together, much like a cowboy rounds up his cattle. By bringing as many pathogens together in the same area, the cells of the immune system can mount an attack and weaken the infection. Other innate immune system cells continue to circulate throughout the body in order to track down any other pathogens that have not been clustered and bound for destruction [12].

The steps of the complement cascade facilitate the search for and removal of antigens by placing them in large clumps, making it easier for other aspects of the immune system to do their jobs. Remember that the complement system is a supplemental cascade of proteins that assists, or "complements" the other aspects of the innate immune system.

The innate immune system works to fight off pathogens before they can start an active infection. For some cases, the innate immune response is not enough, or the pathogen is able to exploit the innate immune response for a way into the host cells. In such situations, the innate immune system works with the adaptive immune system to reduce the severity of infection, and to fight off any additional invaders while the adaptive immune system is busy destroying the initial infection [13].

\section{Conclusion}

Immunology is the study of the immune system and is a very important branch of the medical and biological sciences. From Edward Jenner's pioneering work in the 18th Century that would ultimately lead to vaccination in its modern form (an innovation that has likely saved more lives than any other medical advance), to the many scientific breakthroughs in the 19th and 20th centuries that would lead to, amongst other things, safe organ transplantation, the identification of blood groups, and the now ubiquitous use of monoclonal antibodies throughout science and healthcare, immunology has changed the face of modern medicine. Immunological research continues to extend horizons in our understanding of how to treat significant health issues, with ongoing research efforts in immunotherapy, autoimmune diseases, and vaccines for emerging pathogens, such as Ebola and Covid 19 disease.

\section{Compliance with ethical standards}

\section{Acknowledgments}

Professor Peter Nyarango of the University of Namibia for mentorship.

\section{References}

[1] World Health Organization

[2] Kendall A. Smith. Edward Jenner and the Small Pox Vaccine. Front Immunol. 2011; 2: 21.

[3] Steve M.BlevinsMichael S.Bronze . Robert Koch and the 'golden age' of bacteriology. International Journal of Infectious Diseases Volume 14, Issue 9, September 2010, Pages e744-e751

[4] Owen .Punt. Stranford. Kuby Immunology.Seventh Edition.2013. 
[5] Janeway CA Jr, Travers P, Walport M, et al.. Immunobiology: The Immune System in Health and Disease. 5th edition.2001

[6] Lindsay B. Nicholson. The immune system. Essays Biochem. 2016 Oct 31; 60(3): 275-301

[7] Kenneth Murphy \& Casey Weaver.Janeway's immumnobiology. $9^{\text {th }}$ Edition.2017

[8] Abul K Abbas et al.Basic Immunlology.Functions \& Disorders of the Immune system.Fourth Edition.2014

[9] Marshall, J.S., Warrington, R., Watson, W. et al. An introduction to immunology and immunopathology. Allergy Asthma Clin Immunol 14, 49 (2018)

[10] Segundo Gonzalez, et al Conceptual aspects of self and nonself discrimination. Self Nonself. 2011 Jan-Mar; 2(1): 19-25.

[11] Dani A.C. Heesterbeeka Mathieu L. Angeliera Richard A. Harrisonb Suzan H.M. Rooijakkersa. Complement and Bacterial Infections: From Molecular Mechanisms to Therapeutic Applications. J Innate Immun 2018;10:455-464

[12] Lauren Thau; Edinen Asuka; Kunal Mahajan. Physiology, Opsonization. Copyright (C 2021, StatPearls Publishing LLC.

[13] Eileen Uribe-Querol et al Phagocytosis: Our Current Understanding of a Universal Biological Process. Front. Immunol., 02 June 2020 | https://doi.org/10.3389/fimmu.2020.01066 